\title{
Origin of Weaker Fermi Level Pinning and Localized Interface States at Metal Silicide Schottky Barriers
}

\author{
Zhaofu Zhang , Yuzheng Guo , John Robertson ${ }^{\dagger, *}$ \\ $\dagger$ Department of Engineering, University of Cambridge, Cambridge CB3 OFA, UK \\ $\$$ College of Engineering, Swansea University, Swansea SA1 8EN, United Kingdom
}

\begin{abstract}
The Schottky barriers of transition metal silicides on silicon are characterised by two anomalous features, a face dependence of Schottky barrier heights (SBHs) and a weaker than expected dependence of the SBHs on work function, or 'weaker Fermi level pinning'. Density functional supercell calculations reported here find that these features arise from the occurrence of localized gap states at interfacial coordination defects, in addition to the usual metal-induced gap states (MIGS), and these lead to pinning energies that increase sequentially across the Si gap from $\mathrm{PtSi}_{2}$ to $\mathrm{YbSi}_{2}$. The interfacial gap states vary in shape with face orientation and cause the unusual face-dependent SBHs. The localized interface defect states are a key missing addition to the MIGS model, that are needed to describe fully the interface bonding such as face orientation or coordination defects. This anomalous Fermi level pinning does not reduce gap state densities but could be used to better control SBHs by creating specific configurations with near band-edge pinning energies, thus giving low contact resistances in highly scaled silicon devices or 2D semiconductors.
\end{abstract}

Keywords: Schottky barrier, Fermi level depinning, metal silicide, semiconductor contacts

\section{INTRODUCTION}

As circuit resistances increasingly limit the performance of semiconductor device as their sizes are scaled down in accordance with Moore's law, it has become critical to minimize contact resistances [1-3]. Contact resistances depend greatly on metal-Si interfacial Schottky barriers, and their effect can be reduced by minimizing the Schottky barrier height $(\mathrm{SBH}) \phi_{\mathrm{n}}$. The SBHs are affected by the density of gap states in the semiconductor, which include the intrinsic metal-induced gap states (MIGS) and any defect states near the barrier [4-10]. These states pin the metal Fermi level $\left(\mathrm{E}_{\mathrm{F}}\right)$ at some energy within the gap. The degree of pinning, expressed by a pinning factor $\mathrm{S}=$ $\partial \phi_{\mathrm{n}} / \partial \Phi_{\mathrm{M}}$, where $\Phi_{\mathrm{M}}$ is the metal work function, depends on the density of gap states at $E_{\mathrm{F}}(\mathrm{N})$, via the Cowley-Sze [4] equation,

$$
S=\frac{1}{1+\frac{e^{2} N \delta}{\varepsilon \varepsilon_{0}}}
$$

where $\delta$ is their decay length into semiconductor and $\varepsilon$ is the dielectric constant. $\mathrm{S}$ varies between $\mathrm{S}=0$ (strong pinning or Bardeen limit) and $\mathrm{S}=1$ (weak pinning or Schottky limit)[5-8]. 
There are three recent strategies to minimize contact resistance: to heavily dope the semiconductor so as to reduce the depletion layer at the barrier [11-13], to attenuate the density of MIGS entering the semiconductor by inserting a thin insulating layer [14-18], or to reduce the Fermi level pinning in some way so as to vary the contact's work function to minimize the SBH [19,20]. However, the doping strategy is reaching its limits [13], whereas the inserting an insulating layer also inserts an additional series resistance, so that it is unclear whether this method provides a nett benefit $[1,17]$. Hence, other ways to vary contact resistances are being sought.

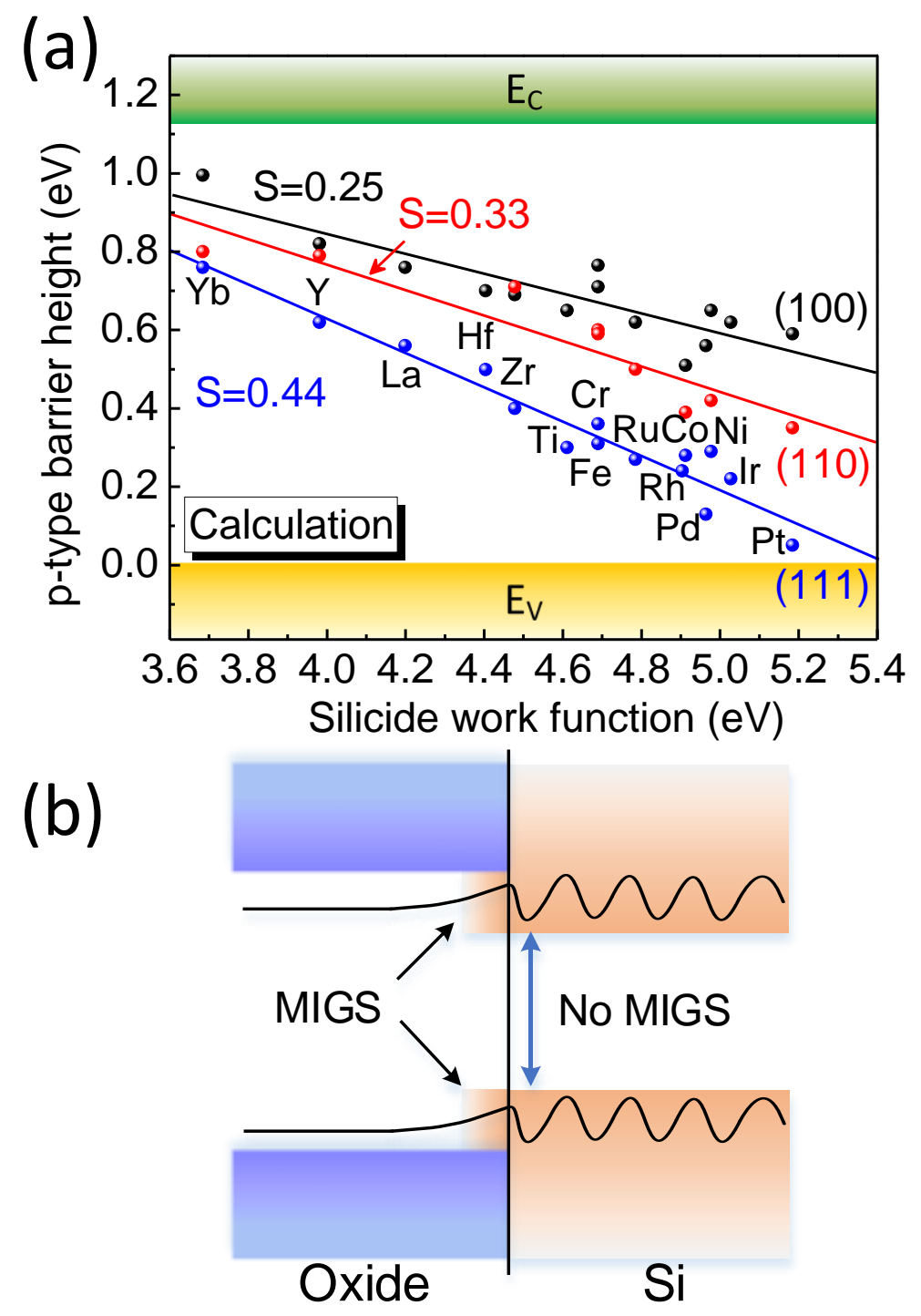

Figure 1. (a) Calculated SBHs of silicides on (111), (100) and (110) Si faces using supercells. (b) Schematic of interface states at oxide/semiconductor interface with type-I alignment, showing MIGS-like states near the oxide band-edges, but none within semiconductor gap.

A possible method is to search more closely into exceptions to the MIGS model of SBHs. Generally, the MIGS model is very successful in describing the chemical trends of $\mathrm{S}$ for a broad range of semiconductors and insulators [5,6]. There are, however, several exceptions to the successes of MIGS theory, such as its failure to explain the apparently weaker pinning factors of metal silicides than elemental metals on Si, as shown in Figure 1(a) [21-23], or germanides [24], the face orientation 
dependence of silicide SBHs [25-28], and the termination dependence of the SBHs of rare-earth arsenides $[29,30]$. These factors have long evaded a simple coherent explanation. This paper explains the unusual behavior of silicide SBHs in terms of interface defect states, additional to the normal MIGS, which cause pinning energies which vary strongly across the Si band gap with the silicide work function and the face orientation.

For reference, a typical version of Fermi level depinning arises for the gate oxides of inversion mode silicon or III-V metal-oxide-semiconductor field-effect transistors (MOSFETs). There, it is necessary to remove any gap states within the semiconductor band gap [31,32], as shown in Figure 1(b), to allow the Fermi level to be swept electrically between the two band edges. This would correspond to a value of $S=1$ within that gap.

Generally, gap states from any nearby point defects increase the density of gap states at $\mathrm{E}_{\mathrm{F}}, \mathrm{N}$, and so would reduce $\mathrm{S}$ in Eq. (1) [4]. Thus, a larger $\mathrm{S}$ for silicides on $\mathrm{Si}$ is apparently beneficial. However, our supercell calculations find that the higher $\mathrm{S}$ value arises from a higher $\mathrm{N}$, so that a different mechanism with extra defect states is occurring. Thus it is not depinning as such. Nevertheless, this process could be beneficial for device contact resistances, if this mechanism can be used to pin $E_{F}$ near a desired band edge to reduce contact resistance - it is not necessary to remove all gap states to achieve this objective.

\section{CALCULATION METHODS}

The calculations use the plane-wave density functional code CASTEP [33], with the generalized gradient approximation (GGA) for the electron exchange-correlation functional, and ultra-soft pseudopotentials with a cutoff energy of $340 \mathrm{eV}$. Energies are converged to below $10^{-6} \mathrm{eV}$ per atom, and forces below $10^{-3} \mathrm{eV} / \AA \hat{\text { A }}$. A k-point mesh of $4 \times 4 \times 1$ is used for Brillouin zone integrations. The SBHs and partial density of states (PDOS) calculations use interface supercells with 9 layers of silicide and 11 layers of $\mathrm{Si}$ with no vacuum layer to follow the interface defect states. And the silicide work functions were obtained by the nonpolar surface supercell with a 30 - $\AA$-thickness vacuum layer.

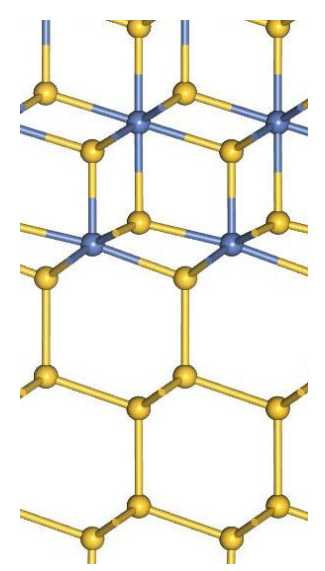

(a) (111)

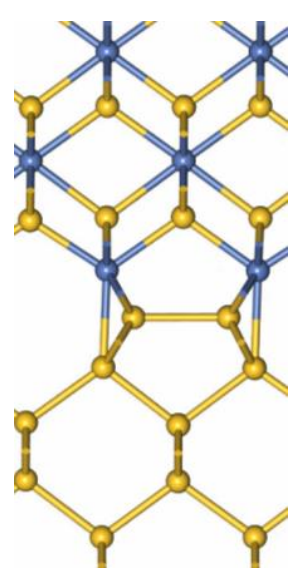

(b) (100)

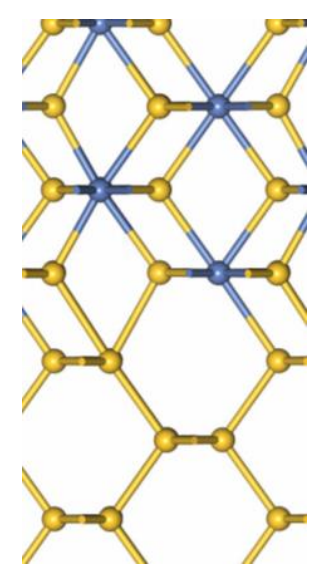

(c) $(110)$

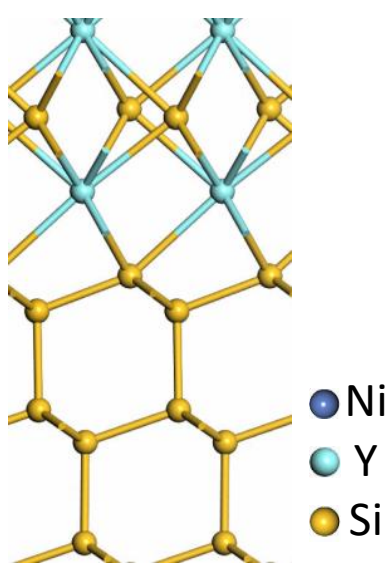

(d) $\mathrm{YSi}_{2} / \mathrm{Si}(111)$

Figure 2. The (a) $1 \times 1$ interface of $\mathrm{NiSi}_{2} / \mathrm{Si}\left(111\right.$ ), (b) $2 \times 1$ dimerized interface of $\mathrm{NiSi}_{2} / \mathrm{Si}(100)$, (c) reconstructed interface of $\mathrm{NiSi}_{2} / \mathrm{Si}(110)$, and (d) $\mathrm{YSi}_{2} / \mathrm{Si}(111)$ interfaces, respectively. 
$\mathrm{NiSi}_{2}$ has a fluorite structure and is well lattice-matched to $\mathrm{Si}$ (mismatch of only $0.1 \%$ ). Its interface structures are highly studied [34,35]. The Si sublattice continues across the interface into the silicide and Si atoms are always 4-fold coordinated. The Ni sites are 8-fold coordinated. At the (111) interface, Ni sites are 7-fold coordinated with a dangling bond, as shown in Figure 2(a).

Some late-transition metals (with higher work function) also have fluorite disilicides, and they make well-matched interfaces with $\mathrm{Si}$, while some have disilicides with different lattices or their silicides with different stoichiometries. For example, the main silicide of $\mathrm{Pd}$ is $\mathrm{Pd}_{2} \mathrm{Si}$ [36]. However, instead we consider a hypothetical cubic $\mathrm{PdSi}_{2}$ and its interfaces with $\mathrm{Si}$. Similarly, $\mathrm{TiSi}_{2}$ is a technologically important silicide. Its C49 or C54 phases have low resistivities, but with moderate lattice-matching with $\mathrm{Si}$ [37]. Again we take a fluorite $\mathrm{TiSi}_{2}$ with a larger lattice constant than $\mathrm{NiSi}_{2}$, but which can be matched to the Si lattice after relaxation. Thus, to find chemical trends, we only focus on metal disilicides in a fluorite structure and their interfaces with $\mathrm{Si}$. We take the (111) interface structure with a 7-fold interfacial metal site and an 'A-type' stacking to follow $\mathrm{NiSi}_{2} / \mathrm{Si}(111)$ [34] even if some like $\mathrm{CoSi}_{2}$ may follow different stackings. Thus from Pt to Ti we focus on the disilicides of their fluorite lattices, and their interfaces with cubic Si. The supercell symmetry and its angles are fixed at $90^{\circ}$ during relaxation to prevent angular distortions. The lattice geometry is relaxed in GGA, and the x,y lattice constants of the silicide are fixed to those of the underlying $\mathrm{Si}$, while allowing the silicide vertical $\mathrm{z}$ distances to relax.

Lanthanide metals have larger atomic radii than $\mathrm{Ni}$ and lower work functions. Their disilicides form a hexagonal layer structure. Their (0001) basal surface is lattice-matched to Si(111) [38]. For typical $\mathrm{YSi}_{2}$, its lattice mismatch is $2.5 \%$ to $\mathrm{Si}$. We take this interfacial structure for other silicides from $\mathrm{ZrSi}_{2}$ to $\mathrm{YbSi}_{2}$ with $\mathrm{Si}$. These disilicide interface models are relaxed like the fluorite disilicides.

We also consider $\operatorname{Si}(100)$ and $\operatorname{Si}(110)$ interfaces. Although, the (100) interfaces of the fluorite disilicides were initially thought to be $1 \times 1$ structures with 6-fold metal sites, high-resolution STEM found that they had a $2 \times 1$ reconstructed geometry with lateral $\mathrm{Si}-\mathrm{Si}$ bonds and 5-fold coordinated $\mathrm{Si}$ sites [39,40], Figure 2(b). The (110) interface for disilicides can be formed by joining (111) and (100)-like interfaces together, as shown in Figure 2(c). The (100)Si/YSi 2 interfaces can be made by turning the $\mathrm{YSi}_{2}$ lattice on one side so that it has nearly the same size as the (100)Si face [23]. Matching for the (110) face can be achieved, but only for a large unit cell. Instead, with some disorder, a good match is found for a smaller cell. Lattice constants of other silicdes may slightly differ from $\mathrm{NiSi}_{2}$ or $\mathrm{YSi}_{2}$, so the interface supercells are forcibly lattice-matched to Si if necessary, and any mismatch is accommodated along the $\mathrm{Oz}$ axis. This roughly conserves the volume of silicides, as $\Phi_{\mathrm{M}}$ is mainly determined by the atomic volume in compound metals.

In this work, we could consider the chemical trends for metal silicides over a wide range of work functions for all three face orientations. For those listed in Figure 1(a), the high work function silicides from $\mathrm{PtSi}_{2}$ to $\mathrm{TiSi}_{2}$ use the $\mathrm{NiSi}_{2}$ fluorite structure, with the interface structure shown in Figure 2(a-c), and the low work function silicides from $\mathrm{YbSi}_{2}$ to $\mathrm{ZrSi}_{2}$ take the $\mathrm{YSi}_{2}$-type hexagonal structure, with the interface structure of Figure 2(d). It should be noted that silicides are a unique class of compounds that span nearly all metals [41-43], perhaps more than any other class of binary compounds. 


\section{RESULTS AND DISCUSSION}

Using the nonpolar surface supercell, the silicide work functions were obtained. The logarithmic fitting gives the silicide work function formula as $\Phi_{\mathrm{MSi} 2}=\Phi_{\mathrm{M}}{ }^{0.44} \Phi_{\mathrm{Si}}{ }^{0.56}$, where $\Phi_{\mathrm{M}}$ is the elemental metal work function [44] and $\Phi_{\mathrm{Si}}$ is the silicon work function, as shown in Figure 3. The SBHs of the different (111), (100) and (110) faces are plotted in Figure 1(a) against the silicide work function. The core-level method is used to derive the SBHs, which is based on the energy difference $(\Delta V)$ between core-level and VBM maintaining constant value in bulk material or interface material $[45,46]$. The $\mathrm{SBH}$ is expressed by [47]:

$$
\Phi_{p}=E_{\text {core }}^{\text {interface }}+\Delta V-E_{F}
$$

where $E_{\text {core }}^{\text {interface }}$ is the core-level state of atoms away from the interface region in the supercell, and $E_{\mathrm{F}}$ is Fermi level.

We see that the resulting SBH values follow a linear trend against the work function for each face, extending previous work [23]. Each face has a different slope S. Interestingly, there is little discontinuity where the silicide lattice type changes from fluorite to $\mathrm{YSi}_{2}$-type.

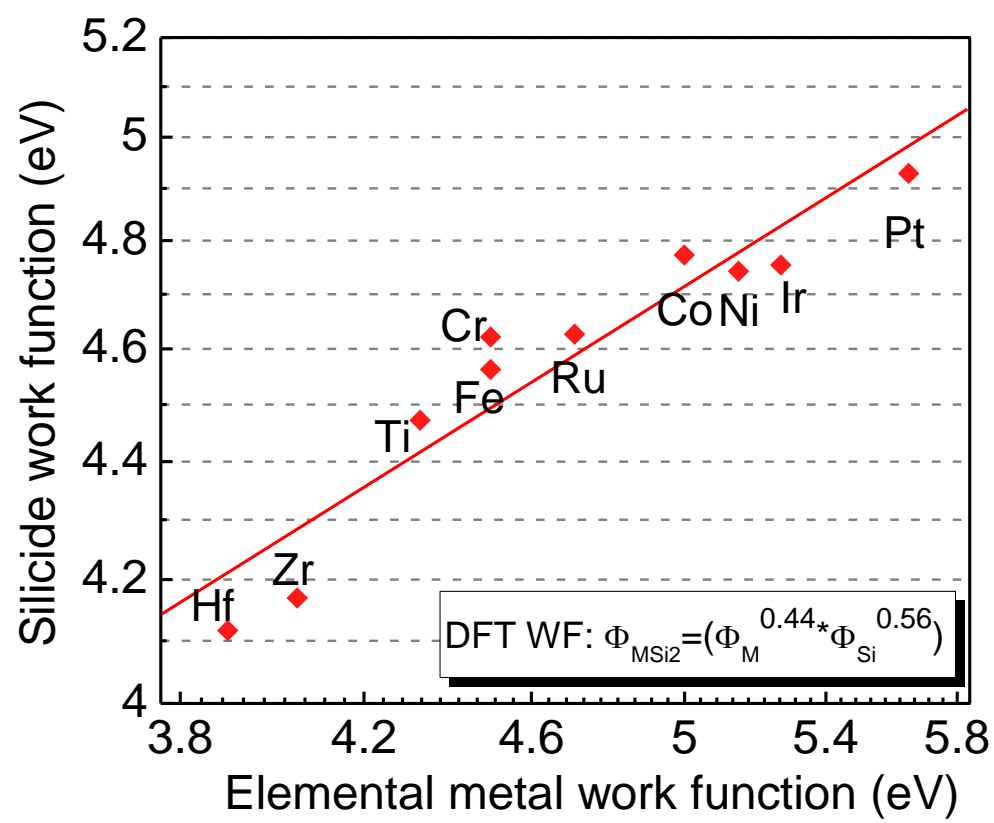

Figure 3. Calculated silicide work function as a function of the elemental metal work function. The logarithmic fitting gives a power of 0.44 for $\Phi_{M}$, as inserted formula.

Thus, a key result in Figure 1(a) is that silicide metals have a much weaker Fermi level pinning on $\mathrm{Si}$, compared to the almost zero slopes of elemental metals [22], and their slopes vary with face. As we noted above, the strong Fermi level pinning of elemental metals on $\mathrm{Si}$ is due to a relatively high density of MIGS. Although MIGS are extensions of metal states, they are actually purely intrinsic semiconductor states, as defined by Tersoff [7]. Hence the MIGS should not vary with the type of metal, either elemental or compound metal (silicide, TiN, etc). Also, for cubic semiconductors, the reference pinning energy or charge neutrality level (CNL) and slope should not vary with face orientation. Thus both factors are contrary to the MIGS model. 
The cause of this effect was not really agreed previously. For $\mathrm{NiSi}_{2} / \mathrm{Si}(111)$ interfaces, the $\mathrm{SBH}$ dependence on face stacking A or B was attributed to the effect of the interfacial Ni dangling bond [23,48-50]. The difference between $\mathrm{Si}(111)$ and (100) face SBHs [26] was larger but was not understood at an atomic level.

Now, although silicides are metallic, their lattices have an underlying covalent skeleton, and their interfaces with Si create an epitaxial array of coordination defects. We notice that the weak pinning and face dependence coincides with the presence of localized interface gap states lying near the Fermi energy $\mathrm{E}_{\mathrm{F}}$ for the $\mathrm{NiSi}_{2} / \mathrm{Si}(111)$ interfaces, as shown in Figure 4. In fact, both properties appear together. Careful examination of the gap states around $\mathrm{E}_{\mathrm{F}}$ finds that they are localized near the interfacial 7-fold Ni site of the (111) face, as seen in Figure 4(b). These defect states are also seen on other silicide (111) interfaces. In each case, they resemble a metal dangling bond.
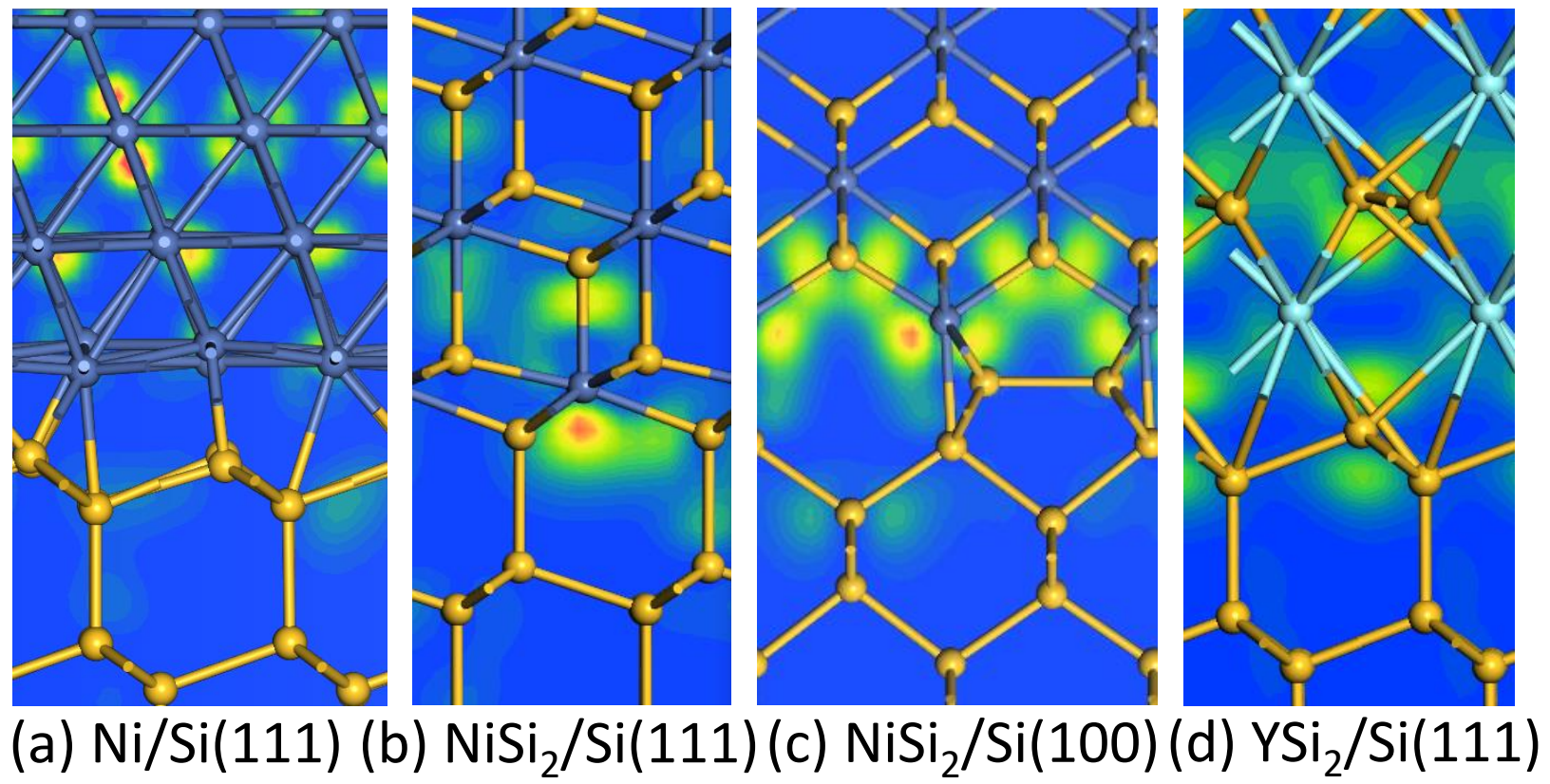

Figure 4. Wavefunction around $\mathrm{E}_{\mathrm{F}}$ for the (a) simple $\mathrm{Ni} / \mathrm{Si}(111)$ interface, (b) $\mathrm{NiSi} / 2 / \mathrm{Si}(111)$ interface, (c) $2 \times 1 \mathrm{NiSi}_{2} / \mathrm{Si}$ (100) interface, and (d) $\mathrm{YSi}_{2} / \mathrm{Si}(111)$ interfaces. The silicides show a state localized in both directions from the interface. MIGS in (a) shows no decay on metal side.

The localized gap states are also found for the $\mathrm{NiSi}_{2}(100)$ interfaces, as seen in Figure 4(c). These defect states are localized around the lateral $\mathrm{Si}$-Si bonds and the 5-fold coordinated $\mathrm{Si}$ sites of the (100) interface. These localized states are also found at other (100) interfaces, localized on the miscoordinated sites. Similar localized gap states are also found at the $\mathrm{YiSi}_{2}(111)$ interface in Figure 4(d).

These interface states have several unusual aspects. They differ from MIGS shown in Figure 4(a) in that they are 'defect-like' in decaying on both the semiconductor side and the metal side of the interface as seen in Figure 4(b). However, they occur for every interface atom, so they are more than a defect. Thus, these interfaces possess three types of states: (1) the travelling wave states on the metal side, (2) the MIGS-like states which are decaying only on the semiconductor side (as in Figure $4 a$ ), and finally (3) the additional 'defect-like' states at the interface, localized in both directions. The third state is a new type and is absent in the existing MIGS model. We find that these states are a 
general feature of systems that show a depinning effect and an orientation dependence of SBH. These anomalous states were seen previously [48-50] but their effects were not studied.

Why do the interfacial defect states occur? The nature of the gap states depends on a variational energy minimization. MIGS are constructed from a basis set of intrinsic VB and CB Si states, which can describe normal MIGS states [7,8]. However, the MIGS basis is insufficiently flexible to describe the metal dangling bond states of (111) or the Si-Si lateral bond or 5-fold Si states of (100) interfaces. For these reasons, a variational calculation causes localized defect states to arise for a more efficient energy minimization.

We have examined the wavefunctions of interface gap states and found that the localized states are grouped as a peak around $E_{F}$. The energy range of these states in (111) and (100) interfaces is shown as the grey peak on each interfacial PDOS in Figure 5. It is strongly peaked and not spread across the whole gap, so it has a high density of states at that energy. In this way, its PDOS is higher than the background MIGS PDOS (which are still present); thus the defect PDOS dominates the pinning property.
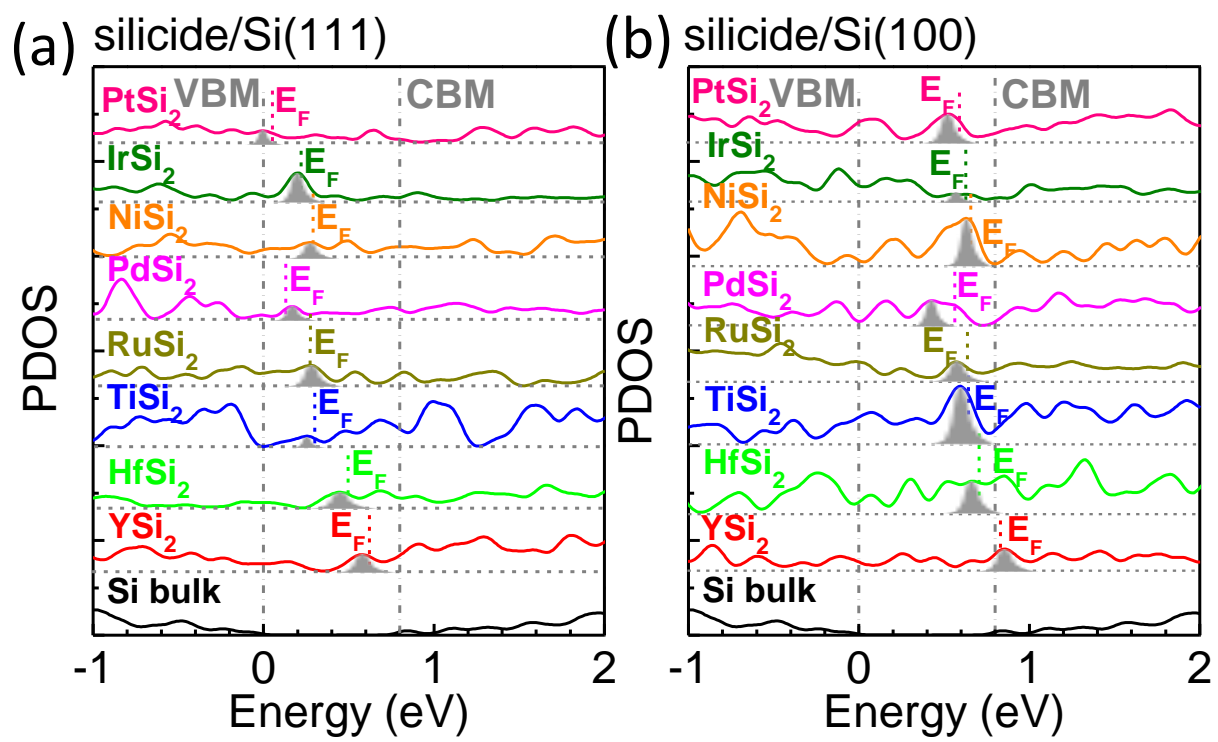

Figure 5. PDOS of the interfacial layer metal silicdes at (a) silicide/Si(111) and (b) silicide/Si(100) supercells. The defect energy is mimicked by a grey shape.

Figure 6 plots the centroid energy of the defect peak for the (111) and (100) faces for each silicide against its work function. The peak moves continuously down in energy as we pass along the Period of silicides, and so its energy scans across the Si gap. Thus the effect of the localized interface states do not depin $\mathrm{E}_{\mathrm{F}}$ as in a classical MIGS picture, but pin $\mathrm{E}_{\mathrm{F}}$ so that it moves step-wise across the gap as the metal changes, one step for each silicide. This sequence follows the well-known variation of metal $d$ orbital energies which decreases across the transition metal series, even as the $d$ levels progressively fill up [51]. In other contexts, this variation allows the tuning of transition metal catalyst properties, and the well-known volcano plot of their catalyst activity $[51,52]$. 


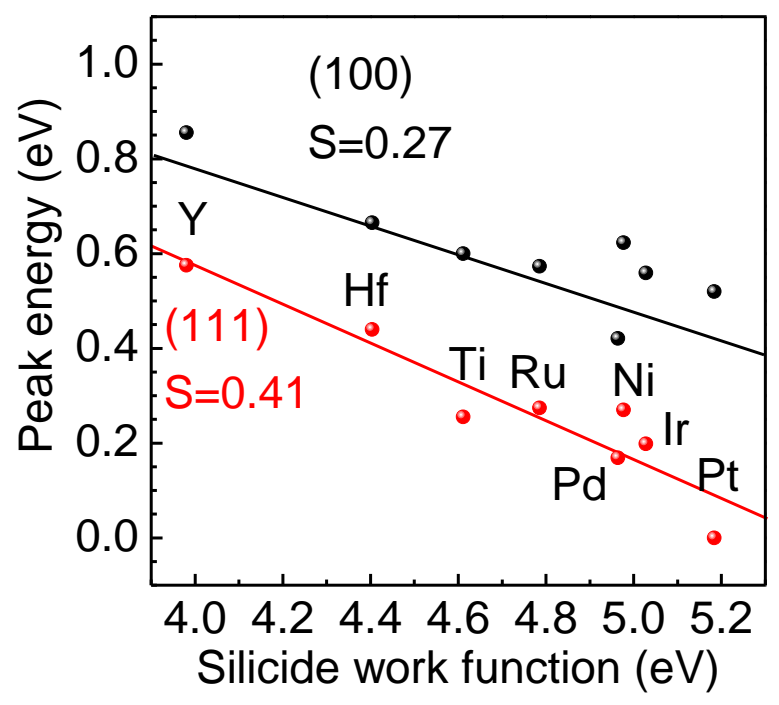

Figure 6. Centroid peak energy against silicide work function for (111) and (001) interfaces.

The localized defect orbital has a different shape for the different face orientations in Figure 4(b,c), so they can have a different pinning energy for each orientation, unlike intrinsic MIGS. This is seen in their different PDOS, Figure 7(a).
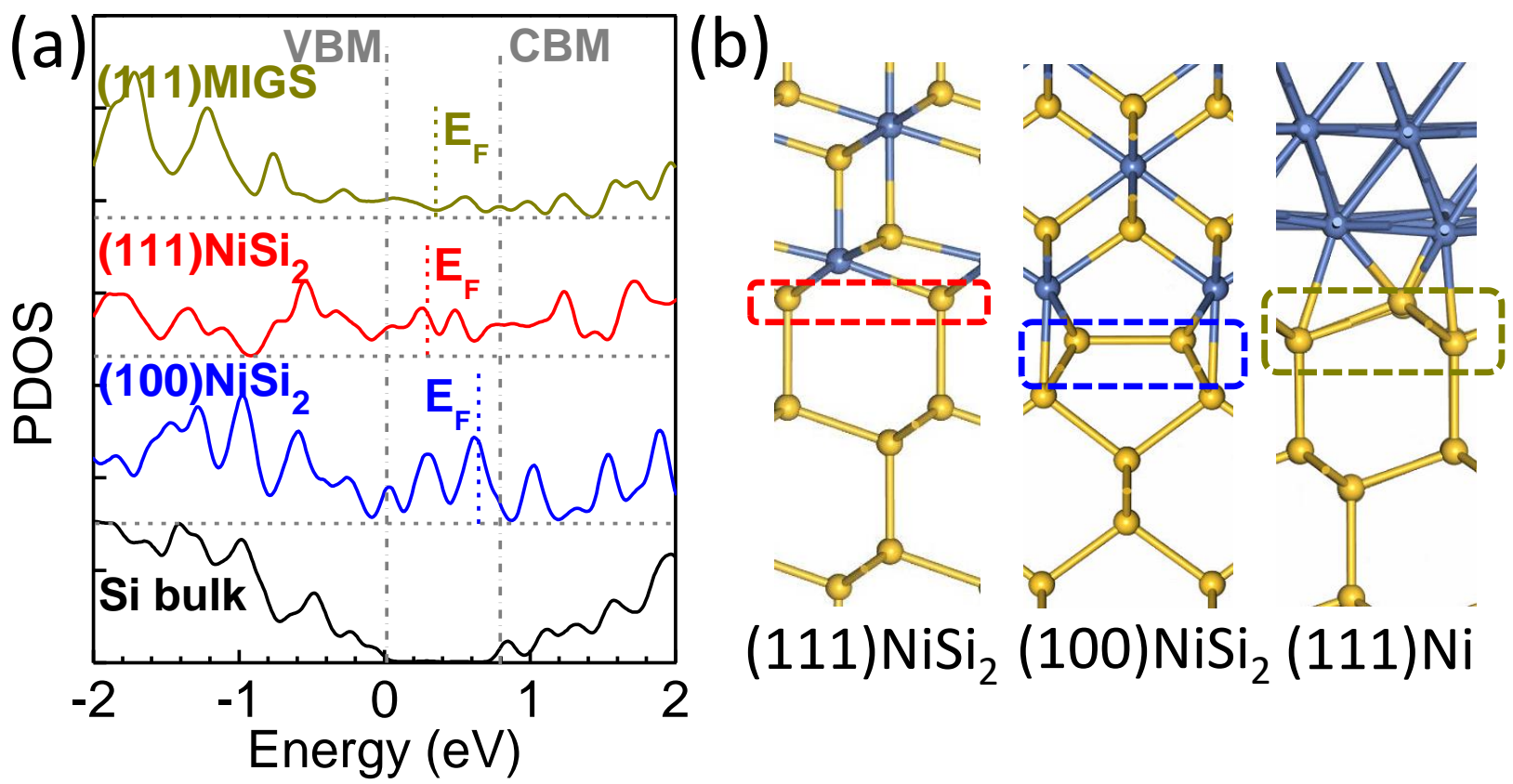

Figure 7. (a) Comparing PDOS of (111) and (100) silicide interfaces showing a higher density of defect states than for simple MIGS. The (111) silicide PDOS peaks at a lower energy than for (100). (b) shows the layer for which the PDOS in (a) is calculated.

The localized gap states occur at mis-coordinated interface sites due to the underlying covalent lattice of silicides failing to fully join the covalent Si lattice. The silicide interfaces create a plane of mis-coordinated sites. Previously, we found that interfaces between metallic rocksalt YAs and semiconducting zincblende GaAs also produce a plane of mis-coordinated sites, and associated localized interface states, so that Ga- and As-terminated interfaces have SBHs that differ by over 1 $\mathrm{eV}$ [30], completely contrary to the MIGS model, but similar to the behavior of silicides. 
The presence of the localized interfacial defect states well explains the sequential variation of pinning energy across the $\mathrm{Si}$ gap for each silicide, at a rate $S$ well above the low slope $S \sim 0$ typical of elemental metals on $\mathrm{Si}$. It also explains the variation of pinning energy with face orientation, because the defect states are anisotropic, unlike MIGS.

Interestingly this mechanism does not cause Fermi level depinning in the sense of a lower N. It creates an additional pinning, which sequentially moves $E_{F}$ across the band gap as the transition metal is changed. These can be seen from a comparison of the typical MIGS PDOS for Si/Ni(111) interfaces and the gap PDOS for the $\mathrm{NiSi}_{2} / \mathrm{Si}(111)$ and $\mathrm{NiSi}_{2} / \mathrm{Si}(100)$ interfaces in Figure 7. The peak doing the pinning for the silicides is higher than that of the MIGS. This is not 'depinning' in that it does not remove all gap states to leave a freely moving Fermi energy. Nevertheless, by selecting the appropriate metal contact, the Fermi level can be pinned at a desired band edge, and can reduce the SBH to near zero with an ohmic contact if a suitable system is found.

\section{CONCLUSION}

In conclusion, we identify metal silicides as lying outside the standard MIGS model with Schottky barrier heights that vary with face orientation and more strongly with work function than elemental metals. The interfaces are found to contain localized interface states due to bonding configurations such as dangling bonds, lateral bonds or over-coordinated sites which are difficult to describe within a MIGS basis set. These states pin the Fermi level at energies moving sequentially across the gap for different silicides. These states represent an addition to the normal MIGS model. These are useful to help design semiconductor interface bonding, where a Fermi-level pinning tunes the SBH and lowers the contact resistances.

\section{ASSOCIATED CONTENT}

\section{AUTHOR INFORMATION}

Corresponding Author

*E-mail: jr@eng.cam.ac.uk

Notes

The authors declare no competing financial interests in this work.

\section{ACKNOWLEDGMENTS}

The authors acknowledge funding from EPSRC grant EP/P005152/1. We also thank Supercomputing Wales for support under Project No. SCW1070. 


\section{REFERENCES}

1. Datta, S.; Pandey, R.; Agrawal, A.; Gupta, S. K.; Arghavani, R. Impact of Contact and Local Interconnect Scaling on Logic Performance. Tech Digest VSLI Technol. 2014, p978.

2. Allain, A.; Kang, J.; Banerjee, K.; Kis, A. Electrical Contacts to Two-Dimensional Semiconductors. Nat. Mater. 2015, 14 (12), 1195-1205.

3. Schulman, D. S.; Arnold, A. J.; Das, S. Contact Engineering for 2D Materials and Devices. Chem. Soc. Rev. 2018, 47, 3037-3058.

4. Cowley, A. M.; Sze, S. M. Surface States and Barrier Height of Metal-Semiconductor Systems. J. Appl. Phys. 1965, 36 (10), 3212-3220.

5. Monch, W. Role of Virtual Gap States and Defects in Metal-Semiconductor Contacts. Phys. Rev. Lett. 1987, 58 (12), 1260-1263.

6. Mönch, W. Chemical Trends of Barrier Heights in Metal-Semiconductor Contacts: On the Theory of the Slope Parameter. Appl. Surf. Sci. 1996, 92, 367-371.

7. Tersoff, J. Schottky Barrier Heights and the Continuum of Gap States. Phys. Rev. Lett. 1984, 52, 465468.

8. Tersoff, J. Schottky Barriers and Semiconductor Band Structures. Phys. Rev. B 1985, 32, 6968-6971.

9. Heine, V. Theory of Surface States. Phys. Rev. 1965, 138, A1689-A1696.

10. Robertson, J. Band Offsets of Wide-Band-Gap Oxides and Implications for Future Electronic Devices. $J$. Vac. Sci. Technol. B 2000, 18 (3), 1785-1791.

11. Rosseel, E.; Profijt, H. B.; Hikavyy, A. Y.; Tolle, J.; Kubicek, S.; Mannaert, G.; L'Abbe, C.; Wostyn, K.; Horiguchi, N.; Clarysse, T. Characterization of Epitaxial Si:C:P and Si:P Layers for Source/Drain Formation in Advanced Bulk FinFETs. ECS Trans. 2014, 64, 977-987.

12. Yu, A. Y. C. Electron Tunneling and Contact Resistance of Metal-Silicon Contact Barriers. Solid State Electron. 1970, 13 (2), 239-247.

13. Weinrich, Z. N.; Li, X.; Sharma, S.; Craciun, V.; Ahmed, M.; Sanchez, E. A. C.; Moffatt, S.; Jones, K. S. Dopant-Defect Interactions in Highly Doped Epitaxial Si:P Thin Films. Thin Solid Films 2019, 685, 1-7.

14. Nishimura, T.; Kita, K.; Toriumi, A. A Significant Shift of Schottky Barrier Heights at Strongly Pinned Metal/Germanium Interface by Inserting an Ultra-Thin Insulating Film. Appl. Phys. Express 2008, 1, 051406.

15. Kobayashi, M.; Kinoshita, A.; Saraswat, K.; Wong, H. S. P.; Nishi, Y. Fermi Level Depinning in metal/Ge Schottky Junction for Metal Source/Drain Ge Metal-Oxide-Semiconductor Field-EffectTransistor Application. J. Appl. Phys. 2009, 105 (2), 023702.

16. Kim, G.; Kim, S.; Park, J.; Han, K. H.; Kim, J.; Yu, H. Schottky Barrier Height Engineering for Electrical Contacts of Multilayered $\mathrm{MoS}_{2}$ Transistors with Reduction of Metal-Induced Gap States. ACS Nano 2018, 12 (6), 6292-6300.

17. Agrawal, A.; Shukla, N.; Ahmed, K.; Datta, S. A Unified Model for Insulator Selection to Form UltraLow Resistivity Metal-Insulator-Semiconductor Contacts to n-Si, n-Ge, and n-InGaAs. Appl. Phys. Lett. 2012, 101 (4), 042108.

18. Schaekers, M.; Barla, K.; Horiguchi, N.; Collaert, N.; Thean, A. V.; De Meyer, K. Contact Resistivities of Metal-Insulator-Semiconductor Contacts and Metal-Semiconductor Contacts. Appl. Phys. Lett. 2016, 108 (17), 171602.

19. Liu, Y.; Guo, J.; Zhu, E.; Liao, L.; Lee, S.; Ding, M.; Shakir, I.; Gambin, V.; Huang, Y.; Duan, X. Approaching the Schottky-Mott Limit in Van der Waals Metal-Semiconductor Junctions. Nature 2018, 557 (7707), 696-700.

20. Wang, Y.; Kim, J. C.; Wu, R. J.; Martinez, J.; Song, X.; Yang, J.; Zhao, F.; Mkhoyan, A.; Jeong, H. Y.; Chhowalla, M. Van der Waals Contacts Between Three-Dimensional Metals and Two-Dimensional Semiconductors. Nature 2019, 568 (7750), 70-74.

21. Freeouf, J. L. Silicide Schottky Barriers: An Elemental Description. Solid State Commun. 1980, 33, 1059-1061.

22. Lin, L.; Guo, Y.; Robertson, J. Metal Silicide Schottky Barriers on Si and Ge Show Weaker Fermi Level Pinning. Appl. Phys. Lett. 2012, 101 (5), 052110.

23. Li, H.; Guo, Y.; Robertson, J. Face Dependence of Schottky Barriers Heights of Silicides and Germanides on Si and Ge. Sci. Rep. 2017, 7 (1), 16669.

24. Nishimura, T.; Yajima, T.; Toriumi, A. Reexamination of Fermi Level Pinning for Controlling Schottky Barrier Height at metal/Ge Interface. Appl. Phys. Express 2016, 9 (8), 081201. 
25. Tung, R. T. Schottky-Barrier Formation at Single-Crystal Metal-Semiconductor Interfaces. Phys. Rev. Lett. 1984, 52, 461-464.

26. Tung, R. T. Schottky Barrier Height-Do we Really Understand What We Measure? J. Vac. Sci. Technol. B 1993, 11, 1546-1552.

27. Tung, R. T. Chemical Bonding and Fermi Level Pinning at Metal-Semiconductor Interfaces. Phys. Rev. Lett. 2000, 84 (26), 6078-6081.

28. Tung, R. T. The Physics and Chemistry of the Schottky Barrier Height. Appl. Phys. Rev. 2014, 1, 011304.

29. Palmstrøm, C. J.; Cheeks, T. L.; Gilchrist, H. L.; Zhu, J. G.; Carter, C. B.; Wilkens, B. J.; Martin, R. Effect of Orientation On the Schottky Barrier Height of Thermodynamically Stable Epitaxial metal/GaAs Structures. J. Vac. Sci. Technol. A 1992, 10 (4), 1946-1953.

30. Zhang, Z.; Guo, Y.; Robertson, J. Termination-Dependence of Fermi Level Pinning at Rare-Earth arsenide/GaAs Interfaces. Appl. Phys. Lett. 2020, 116 (25), 251602.

31. Tersoff, J. Theory of Semiconductor Heterojunctions: The Role of Quantum Dipoles. Phys. Rev. B 1984, 30, 4874-4877.

32. Robertson, J. Model of Interface States at III-V Oxide Interfaces. Appl. Phys. Lett. 2009, 94 (15), 152104.

33. Clark, S. J.; Segall, M. D.; Pickard, C. J.; Hasnip, P. J.; Probert, M. J.; Refson, K.; Payne, M. C. First Principles Methods Using CASTEP. Z. Kristallogr. 2005, 220, 567-570.

34. Cherns, D.; Anstis, G. R.; Hutchison, J. L.; Spence, J. C. H. Atomic Structure of the NiSi $2 /(111) \mathrm{Si}$ Interface. Philos. Mag. A 1982, 46 (5), 849-862.

35. Cherns, D.; Hetherington, C. J. D.; Humphreys, C. J. The Atomic Structure of the $\mathrm{NiSi}_{2}-(001) \mathrm{Si}$ Interface. Philos. Mag. A 1984, 49 (1), 165-177.

36. Ho, P. S.; Schmid, P. E.; Föll, H. Stoichiometric and Structural Origin of Electronic States at the $\mathrm{Pd}_{2} \mathrm{Si}-$ Si. Phys. Rev. Lett. 1981, 46, 782-785.

37. Jeon, H.; Sukow, C. A.; Honeycutt, J. W.; Rozgonyi, G. A.; Nemanich, R. J. Morphology and Phase Stability of $\mathrm{TiSi}_{2}$ on Si. J. Appl. Phys. 1992, 71 (9), 4269-4276.

38. Vandré, S.; Kalka, T.; Preinesberger, C.; Dähne-Prietsch, M. Flatband Conditions Observed for Lanthanide-Silicide Monolayers On n-Type Si(111). Phys. Rev. Lett. 1999, 82, 1927-1930.

39. Yu, B. D.; Miyamoto, Y.; Sugino, O.; Sakai, A.; Sasaki, T.; Ohno, T. Structural and Electronic Properties of Metal-Silicide/Silicon Interfaces: A First principles Study. J. Vac. Sci. Technol. B 2001, 19, 1180-1185.

40. Falke, U.; Bleloch, A.; Falke, M.; Teichert, S. Atomic Structure of a $(2 \times 1)$ Reconstructed NiSi $/ 2 i(001)$ Interface. Phys. Rev. Lett. 2004, 92 (11), 116103.

41. Meschel, S. V.; Kleppa, O. J. Standard Enthalpies of Formation of some 3d Transition Metal Silicides by High Temperature Direct Synthesis Calorimetry. J. Alloy. Compd. 1998, 267 (1), 128-135.

42. Meschel, S. V.; Kleppa, O. J. Standard Enthalpies of Formation of some 4d Transition Metal Silicides by High Temperature Direct Synthesis Calorimetry. J. Alloy. Compd. 1998, 274 (1), 193-200.

43. Meschel, S. V.; Kleppa, O. J. Standard Enthalpies of Formation of some 5d Transition Metal Silicides by High Temperature Direct Synthesis Calorimetry. J. Alloy. Compd. 1998, 280 (1), 231-239.

44. Michaelson, H. B. The Work Function of the Elements and its Periodicity. J. Appl. Phys. 1977, 48 (11), 4729-4733.

45. Kraut, E. A.; Grant, R. W.; Waldrop, J. R.; Kowalczyk, S. P. Precise Determination of the ValenceBand Edge in X-Ray Photoemission Spectra: Application to Measurement of Semiconductor Interface Potentials. Phys. Rev. Lett. 1980, 44 (24), 1620-1623.

46. Zhang, Z.; Guo, Y.; Robertson, J. Chemical Bonding and Band Alignment at $\mathrm{X}_{2} \mathrm{O}_{3} / \mathrm{GaN}(\mathrm{X}=\mathrm{Al}, \mathrm{Sc})$ Interfaces. Appl. Phys. Lett. 2019, 114 (16), 161601.

47. Zhang, Z.; Guo, Y.; Robertson, J. Phase Dependence of Schottky Barrier Heights for Ge-Sb-Te and Related Phase-Change Materials. J. Appl. Phys. 2020, 127 (15), 155301.

48. Das, G. P.; Blochl, P.; Andersen, O. K.; Christensen, N. E.; Gunnarsson, O. Electronic Structure and Schottky-barrier Heights of (111) $\mathrm{NiSi}_{2} / \mathrm{Si} \mathrm{A}$ - and B-type Interfaces. Phys. Rev. Lett. 1989, 63 (11), 1168-1171.

49. Ossicini, S.; Bisi, O.; Bertoni, C. M. Electronic Structure of Si(111)-NiSi 2 (111) A-type and B-type Interfaces. Phy.s Rev. B 1990, 42 (9), 5735-5743. 
50. Fujitani, H.; Asano, S. Schottky-Barrier Height and Electronic Structure of the Si Interface with Metal Silicides: $\mathrm{CoSi}_{2}, \mathrm{NiSi}_{2}$, and $\mathrm{YSi}_{2}$. Phys. Rev. B 1994, 50, 8681-8698.

51. Hammer, B.; Morikawa, Y.; Norskov, J. K. CO Chemisorption at Metal Surfaces and Overlayers. Phys. Rev. Lett. 1996, 76 (12), 2141-2144.

52. Robertson, J. Heterogeneous Catalysis Model of Growth Mechanisms of Carbon Nanotubes, Graphene, and Silicon Nanowires. J. Mater. Chem. 2012, 22, 19858-19862.

TOC Graphic:

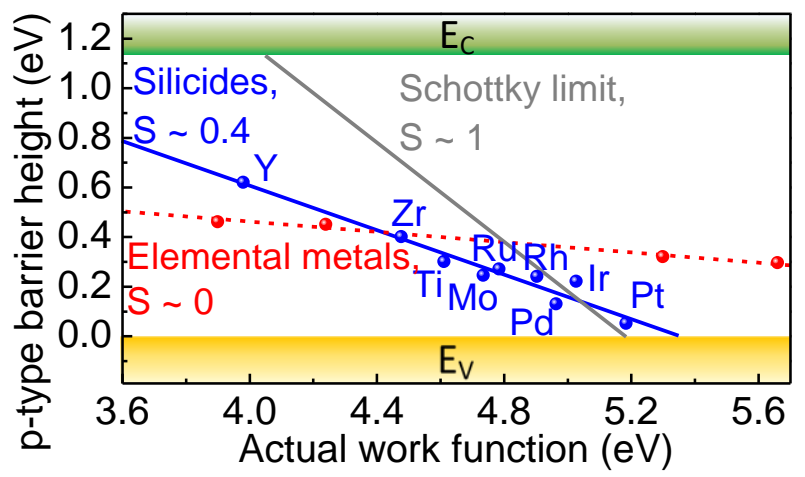

